\title{
Existence and data-dependence theorems for fractional impulsive integro-differential system
}

\author{
Hasib Khan ${ }^{1}$, Zareen A. Khan ${ }^{2 *}$, Haleh Tajadodi ${ }^{3}$ and Aziz Khan ${ }^{4}$
}

\author{
"Correspondence: \\ dr.zareenkhan@ymail.com \\ ${ }^{2}$ Department of Mathematics, \\ College of Science, Princess Nourah \\ bint Abdulrahman University, \\ Riyadh, Saudi Arabia \\ Full list of author information is \\ available at the end of the article
}

\begin{abstract}
In this article we have considered a fractional order impulsive integro-differential equation (IDE) in Caputo's sense for the unique solution and data dependence results. We take help of the Banach fixed point theory and basic literature of fractional calculus. The results are examined with the help of an expressive numerical example for an application of the results.
\end{abstract}

MSC: Primary 26A33; secondary 34A12; 34K40; 47H08

Keywords: Fractional impulsive IDE; Banach fixed point theorem; Stability analysis; Uniqueness of solution

\section{Introduction}

Modeling with the help of fractional order integral and differential operators is very common in the community of engineers and scientists due to the applications. Recently, experts of theory and numeric methods have given interesting tools for the study of fractional order models. In the theoretical aspects of the models, fixed point theorems play a vital role. We suggest the readers for more detail about the fractional calculus and its application to the work in [1-7]. Among the fractional operators, the Caputo-Fabrizio [8-10] and the Atangana-Baleanu fractional differential operators with nonsingular kernel $[4-7,11-15]$ are recently well studied operators.

Recently, some researchers have focused on the different types of FDEs with impulses for the existence of solutions (EUS). Here, we highlight some of them. Sousa et al. [16] considered the investigation of existence results and Ulam-stability by the help of fixed point approach of an impulsive system. Xu and Liu [17] studied the boundedness criteria for delay impulsive system and provided an application. Zhang and Xiong [18] used some properties of the Mittag-Leffler function with one and two parameters for the existence and stability results. Zhao et al. [19] evaluated fractional order impulsive systems with Dirichlet boundaries by the help of Morse theory for the EUS. Heidarkhani et al. [20] investigated multiple solutions with the help of three critical points approach.

For the application of the IDEs with impulses, we recommend the readers the recent work [21-23]. Keeping the importance of the study, we are considering the following im-

(c) The Author(s) 2020. This article is licensed under a Creative Commons Attribution 4.0 International License, which permits use, sharing, adaptation, distribution and reproduction in any medium or format, as long as you give appropriate credit to the original author(s) and the source, provide a link to the Creative Commons licence, and indicate if changes were made. The images or other third party material in this article are included in the article's Creative Commons licence, unless indicated otherwise in a credit line to the material. If material is not included in the article's Creative Commons licence and your intended use is not permitted by statutory regulation or exceeds the permitted use, you will need to obtain permission directly from the copyright holder. To view a copy of this licence, visit http://creativecommons.org/licenses/by/4.0/. 
pulsive IDE for the existence, stability, and numerical solution:

$$
\left\{\begin{array}{l}
{ }^{c} \mathcal{D}^{\vartheta} x(t)=\Psi(t, x(t))+\int_{a}^{t} \mathcal{K}_{0}(t, s, x(s)) d s, \\
x(t)=\frac{1}{\Gamma(\vartheta)} \int_{t_{k}}^{t}(t-s)^{\vartheta-1} \mathcal{G}_{k}\left(s, x\left(s_{k}^{-}\right)\right) d s, \quad t \in\left(t_{k}, \delta_{k}\right], \\
\beta_{1} x(0)+\beta_{2} x(b)=\kappa(0),
\end{array}\right.
$$

where $\Psi:[a, b] \times \mathbb{R} \rightarrow \mathbb{R}, \mathcal{K}_{0}:[a, b] \times[a, b] \times \mathbb{R} \rightarrow \mathbb{R}, \Psi:[a, b] \rightarrow \mathbb{R}$ are continuous functions in the arguments with $\left.\Psi(t, x(t))\right|_{t=0}=0$. The ${ }_{a}^{c} \mathcal{D}^{\vartheta}$ is Caputo's differential operator of order $\vartheta \in(0,1]$. We consider the split of the interval $[a, b]$ with respect to $t_{k}$, $\delta_{k}$ such that $a<t_{k}<\delta_{k}<b$ for $k=1,2,3, \ldots, m$ and assume $\delta_{m+1}=b$. We consider Banach space $C^{1}\left([0, b], \mathbb{R}^{n}\right)$ of all the continuous functions with norm $\|x\|_{\infty}=\sup _{t \in[0, b]}\|x(t)\|$, where $\|\cdot\|$ is a complete norm in $\mathbb{R}^{n}$, where $C^{1}([0, b])(\mathcal{M})=\left\{x \in C^{1}\left([0, b], \mathbb{R}^{n}\right):\|x\|_{\infty} \leq\right.$ $\mathcal{M}$ for all $\mathcal{M}>0\}$.

Definition 1.1 Fractional order integral of $\zeta:(0,+\infty) \rightarrow \mathbb{R}$ for order $\kappa>0$ is

$$
\mathcal{I}_{0}^{\kappa} \zeta(t)=\frac{1}{\Gamma(\kappa)} \int_{0}^{t}(t-s)^{\kappa-1} \zeta(s) d s
$$

such that the integral is defined on $(0,+\infty)$, where

$$
\Gamma(\kappa)=\int_{0}^{+\infty} e^{-s} s^{\kappa-1} d s
$$

Definition 1.2 For a fractional order $\kappa>0$, Caputo's derivative for $\zeta(t):(0,+\infty) \rightarrow \mathbb{R}$ is given by

$$
\mathcal{D}^{\kappa} \zeta(t)=\frac{1}{\Gamma(k-\kappa)} \int_{0}^{t}(t-s)^{k-\kappa-1} \zeta^{(k)}(s) d s
$$

for $k=[\kappa]+1$, where $[\kappa]$ is used for the integer part of $\kappa$.

\section{Integral form}

This section is reserved for the integral form of fractional order impulsive system (1.1)

Theorem 2.1 For $\vartheta \in(0,1]$ and $\mathbb{H}(t, x(t)) \in C[a, b]$ such that $x(t)$ is a solution of

$$
\left\{\begin{array}{l}
{ }_{a}^{c} \mathcal{D}^{\vartheta} x(t)=\mathbb{H}(t, x(t)), \\
x(t)=\frac{1}{\Gamma(\vartheta)} \int_{t_{k}}^{t}(t-s)^{\vartheta-1} \mathcal{G}_{k}\left(s, x\left(s_{k}^{-}\right)\right) d s, \quad t \in\left(t_{k}, \delta_{k}\right], \\
\beta_{1} x(0)+\beta_{2} x(b)=\kappa(0),
\end{array}\right.
$$


provided that

$$
x(t)=\left\{\begin{array}{l}
\frac{\kappa(0)}{\beta_{1}}-\frac{\beta_{2}}{\beta_{1} \Gamma(\vartheta)}\left[\int_{t_{k}}^{\delta_{k}}\left(\delta_{k}-s\right)^{\vartheta-1} \mathcal{G}_{k}\left(s, x\left(s_{k}^{-}\right)\right) d s+\int_{t_{k}}^{b}(b-s)^{\vartheta-1} \mathbb{H}(s, x(s)) d s\right] \\
\quad+\frac{1}{\Gamma(\vartheta)} \int_{0}^{t}(t-s)^{\vartheta-1} \mathbb{H}(s, x(s)) d s, \\
\quad \text { for } t \in\left[0, t_{1}\right], \\
\frac{1}{\Gamma(\vartheta)} \int_{t_{k}}^{t}(t-s)^{\vartheta-1} \mathcal{G}_{k}\left(s, x\left(s_{k}^{-}\right)\right) d s, \\
\quad \text { for } t \in\left[t_{k}, \delta_{k}\right] \\
\vdots \\
\frac{1}{\Gamma(\vartheta)} \int_{t_{k}}^{\delta_{k}}\left(\delta_{k}-s\right)^{\vartheta-1} \mathcal{G}_{k}\left(s, x\left(s_{k}^{-}\right)\right) d s+\frac{1}{\Gamma(\vartheta)} \int_{t_{k}}^{t}(t-s)^{\vartheta-1} \mathbb{H}(s, x(s)) d s, \\
\quad \text { for } t \in\left[\delta_{k}, t_{k+1}\right] .
\end{array}\right.
$$

Proof We divide the proof in parts as follows.

Case-I For $t \in\left(0, t_{1}\right]$, applying the integral operator $\mathcal{I}^{\vartheta}$ on $(2.1)$, we have

$$
x(t)=x(0)+\frac{1}{\Gamma(\vartheta)} \int_{0}^{t}(t-s)^{\vartheta-1} \mathbb{H}(s, x(s)) d s .
$$

Case-II For $t \in\left(\delta_{k}, t_{k+1}\right]$, applying the integral operator $\mathcal{I}^{\vartheta}$ on (2.1), we have

$$
x(t)=x\left(\delta_{k}\right)+\frac{1}{\Gamma(\vartheta)} \int_{t_{k}}^{t}(t-s)^{\vartheta-1} \mathbb{H}(s, x(s)) d s,
$$

where by the help of the impulsive relation

$$
x(t)=\frac{1}{\Gamma(\vartheta)} \int_{t_{k}}^{t}(t-s)^{\vartheta-1} \mathcal{G}_{k}\left(s, x\left(s_{k}^{-}\right)\right) d s
$$

we get

$$
x\left(\delta_{k}\right)=\frac{1}{\Gamma(\vartheta)} \int_{t_{k}}^{\delta_{k}}\left(\delta_{k}-s\right)^{\vartheta-1} \mathcal{G}_{k}\left(s, x\left(s_{k}^{-}\right)\right) d s
$$

Thus, (2.4) implies

$$
x(t)=\frac{1}{\Gamma(\vartheta)} \int_{t_{k}}^{\delta_{k}}\left(\delta_{k}-s\right)^{\vartheta-1} \mathcal{G}_{k}\left(s, x\left(s_{k}^{-}\right)\right) d s+\frac{1}{\Gamma(\vartheta)} \int_{t_{k}}^{t}(t-s)^{\vartheta-1} \mathbb{H}(s, x(s)) d s .
$$

Now, using the condition $\beta_{1} x(0)+\beta_{2} x(t)=\kappa(0)$, we have

$$
x(0)=\frac{\kappa(0)}{\beta_{1}}-\frac{\beta_{2}}{\beta_{1} \Gamma(\vartheta)}\left[\int_{t_{k}}^{\delta_{k}}\left(\delta_{k}-s\right)^{\vartheta-1} \mathcal{G}_{k}\left(s, x\left(s_{k}^{-}\right)\right) d s+\int_{t_{k}}^{b}(b-s)^{\vartheta-1} \mathbb{H}(s, x(s)) d s\right] .
$$

Thus, by the help of (2.3) and (2.8), for $t \in\left[0, t_{1}\right]$, we have

$$
\begin{aligned}
x(t)= & \frac{\kappa(0)}{\beta_{1}}-\frac{\beta_{2}}{\beta_{1} \Gamma(\vartheta)}\left[\int_{t_{k}}^{\delta_{k}}\left(\delta_{k}-s\right)^{\vartheta-1} \mathcal{G}_{k}\left(s, x\left(s_{k}^{-}\right)\right) d s+\int_{t_{k}}^{b}(b-s)^{\vartheta-1} \mathbb{H}(s, x(s)) d s\right] \\
& +\frac{1}{\Gamma(\vartheta)} \int_{0}^{t}(t-s)^{\vartheta-1} \mathbb{H}(s, x(s)) d s .
\end{aligned}
$$


Case-III For $t \in\left(t_{k}, \delta_{k}\right]$, we have

$$
x(t)=\frac{1}{\Gamma(\vartheta)} \int_{t_{k}}^{t}(t-s)^{\vartheta-1} \mathcal{G}_{k}\left(s, x\left(s_{k}^{-}\right)\right) d s .
$$

This completes the proof.

Corollary 2.1 By replacing $\mathbb{H}(t, x(t))$ with $\Psi(t, x(t))+\int_{a}^{t} \mathcal{K}_{0}(t, s, x(s))$ ds, while keeping the conditions and order of derivative the same as in the theorem above, we get the following solution for fractional impulsive system (1.1):

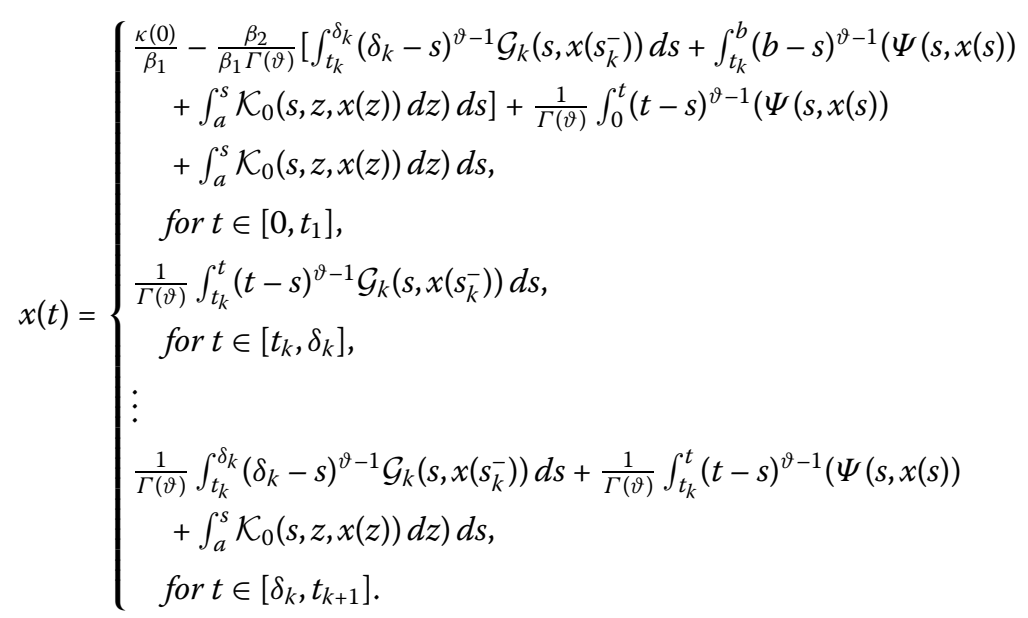

\section{Theorems for EUS}

For the main results of this manuscript, we convert the suggested problem (1.1) into a fixed point problem. For this, we introduce the following operator:

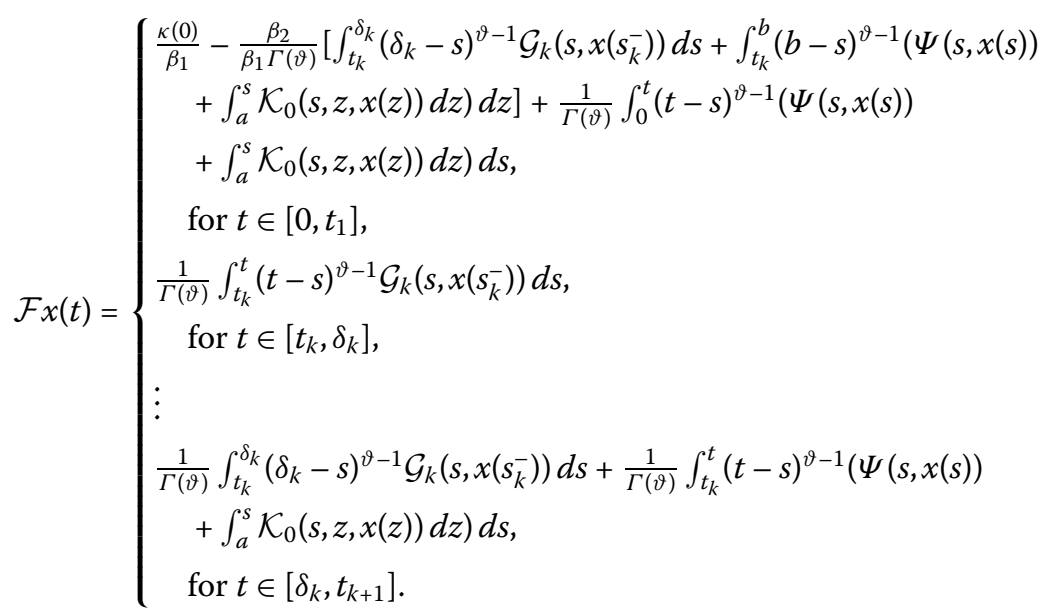

The following assumptions are assumed for the proof of our main results:

$\left(\mathcal{A}_{1}\right) \Psi(t, x(t)):[0, b] \times \mathbb{R} \rightarrow \mathbb{R}, \mathcal{K}_{0}(t, s, x(t)):[0, b] \times[0, b] \times \mathbb{R} \rightarrow \mathbb{R}$ are continuous functions, and there exist constants $\mathbb{K}_{1}, \mathbb{K}_{2}, \mathbb{K}_{3}, \mathbb{K}_{4}, \mathbb{K}_{5}, \mathbb{K}_{6} \in \mathbb{R}$ such that

1. $|\Psi(t, x(t))-\Psi(t, y(t))| \leq \mathbb{K}_{1}|x(t)-y(t)|$ for all $t \in[0, b]$ and $x, y \in \mathbb{R}$;

2. $|\Psi(t, x(t))| \leq \mathbb{K}_{2}+\mathbb{K}_{3}|x(t)|$;

3. $\left|\mathcal{K}_{0}(t, s, x(t))-\mathcal{K}_{0}(t, s, y(t))\right| \leq \mathbb{K}_{4}|x(t)-y(t)|$ for all $t, s \in[0, b]$ and $x, y \in \mathbb{R}$; 
4. $\left|\mathcal{K}_{0}(t, s, x(t))\right| \leq \mathbb{K}_{5}+\mathbb{K}_{6}|x(t)|$.

$\left(\mathcal{A}_{2}\right) \mathcal{G}_{k}: I_{k} \times \mathbb{R} \rightarrow \mathbb{R}$ for $I_{k}=\left[t_{k}, \delta_{k}\right]$, for $k=1,2, \ldots, p$ are continuous and there exist positive constants $\mathbb{K}_{g}, \mathbb{L}_{g}$ such that

1. $\left|\mathcal{G}_{k}(t, x(t))-\mathcal{G}_{k}(t, y(t))\right| \leq \mathbb{K}_{g}|x-y|$ for all $x, y \in \mathbb{R}, t \in I_{k}, k=1,2, \ldots, p$;

2. $\left|\mathcal{G}_{k}(t, x(t))\right| \leq \mathbb{L}_{g}$ for all $t \in I_{k}$ and $x(t) \in \mathbb{R}$.

$\left(A_{3}\right)$ For $\mathcal{M}=\|x(t)\|, \chi_{1}=\frac{\kappa(0)}{\beta_{1}}+\frac{b^{\vartheta} \beta_{2} \mathbb{L}_{g}}{\beta_{1} \Gamma(\vartheta+1)}+\left(\frac{b^{\vartheta} \beta_{2}}{\beta_{1} \Gamma(\vartheta+1)}+\frac{b^{\vartheta}}{\Gamma(\vartheta+1)}\right)\left(\mathbb{K}_{2}+\mathbb{K}_{3} \mathcal{M}+\mathbb{K}_{5}+\right.$ $\left.\mathbb{K}_{6} \mathcal{M}\right), \frac{1}{\Gamma(\vartheta+1)} \mathbb{L}_{g} b^{\vartheta}+\frac{1}{\Gamma(\vartheta+1)} b^{\vartheta}\left(\mathbb{K}_{2}+\mathbb{K}_{3} \mathcal{M}+\left(\mathbb{K}_{5}+\mathbb{K}_{6} \mathcal{M}\right)\right), \frac{\mathbb{L}_{g}}{\Gamma(\vartheta+1)} b^{\vartheta}$, and $\chi=$ $\max \left\{\chi_{1}, \chi_{2}, \chi_{3}\right\}$.

$\left(A_{4}\right)$ For real values $\mathbb{K}_{i}$ for $i=1,2,3,4, t, s \in[0, b],\|x(t)\|=\mathcal{M}$ with $\zeta=\max \left\{\frac{\|\kappa(0)-\widetilde{\kappa}(0)\|}{\beta_{1}}+\right.$ $\left.\frac{\beta_{2}\left[b^{\vartheta} \mathbb{K}_{g}+b^{\vartheta}\left(\mathbb{K}_{1}+b \mathbb{K}_{4}\right)\right]+\beta_{1} b^{\vartheta}\left(\mathbb{K}_{1}+(s-a) \mathbb{K}_{4}\right)}{\beta_{1} \Gamma(\vartheta+1)}, \frac{b^{\vartheta}}{\Gamma(\vartheta+1)}\left[\mathbb{K}_{g}+\left(\mathbb{K}_{1}+b \mathbb{K}_{4}\right)\right], \frac{1}{\Gamma(\vartheta+1)} b^{\vartheta} \mathbb{K}_{g}\right\}\|x-\tilde{x}\|$.

Theorem 3.1 Assume that conditions $\left(\mathcal{A}_{1}\right)-\left(\mathcal{A}_{3}\right)$ are satisfied, then the fractional IDE (1.1) has a unique solution provided that $\zeta<1$.

Proof For $\mathbb{B}=\{x(t) \in P C([0, b], \mathbb{R}):\|x\| \leq \mathcal{M}\}$, we prove that the operator $\mathcal{T}: \mathbb{B} \rightarrow \mathbb{B}$.

For the unique fixed point of the operator $\mathcal{F}$, we divide the proof in the following three steps.

Step 1. For $t \in\left[0, t_{1}\right],(3.1)$ implies

$$
\begin{aligned}
\|\mathcal{T} x(t)\|= & \| \frac{\kappa(0)}{\beta_{1}}-\frac{\beta_{2}}{\beta_{1}}\left[\frac{1}{\Gamma(\vartheta)} \int_{t_{k}}^{\delta_{k}}\left(\delta_{k}-s\right)^{\vartheta-1} \mathcal{G}_{k}\left(s, x\left(s_{k}^{-}\right)\right) d s\right. \\
& \left.+\frac{1}{\Gamma(\vartheta)} \int_{t_{k}}^{b}(b-s)^{\vartheta-1}\left(\Psi(s, x(s))+\int_{a}^{s} \mathcal{K}_{0}(s, z, x(z)) d z\right) d z\right] \\
& +\frac{1}{\Gamma(\vartheta)} \int_{0}^{t}(t-s)^{\vartheta-1}\left(\Psi(s, x(s))+\int_{a}^{s} \mathcal{K}_{0}(s, z, x(z)) d z\right) d s \| \\
\leq & \left\|\frac{\kappa(0)}{\beta_{1}}\right\|+\frac{\beta_{2}}{\beta_{1}}\left[\frac{1}{\Gamma(\vartheta)} \int_{t_{k}}^{\delta_{k}}\left(\delta_{k}-s\right)^{\vartheta-1}\left\|\mathcal{G}_{k}\left(s, x\left(s_{k}^{-}\right)\right)\right\| d s\right. \\
& \left.+\frac{1}{\Gamma(\vartheta)} \int_{t_{k}}^{b}(b-s)^{\vartheta-1}\left(\|\Psi(s, x(s))\|+\int_{a}^{s}\left\|\mathcal{K}_{0}(s, z, x(z))\right\| d z\right) d z\right] \\
& +\frac{1}{\Gamma(\vartheta)} \int_{0}^{t}(t-s)^{\vartheta-1}\left(\|\Psi(s, x(s))\|+\int_{a}^{s}\left\|\mathcal{K}_{0}(s, z, x(z))\right\| d z\right) d s \\
\leq & \frac{\kappa(0)}{\beta_{1}}+\frac{\beta_{2}}{\beta_{1}}\left[\frac{\mathbb{L}_{g}}{\Gamma(\vartheta)} \int_{t_{k}}^{\delta_{k}}\left(\delta_{k}-s\right)^{\vartheta-1} d s\right. \\
& \left.+\frac{1}{\Gamma(\vartheta)} \int_{t_{k}}^{b}(b-s)^{\vartheta-1}\left(\mathbb{K}_{2}+\mathbb{K}_{3}|x(t)|+\int_{a}^{s}\left(\mathbb{K}_{5}+\mathbb{K}_{6}|x(t)|\right) d z\right) d z\right] \\
& +\frac{1}{\Gamma(\vartheta)} \int_{0}^{t}(t-s)^{\vartheta-1}\left(\mathbb{K}_{2}+\mathbb{K}_{3}|x(t)|+\int_{a}^{s}\left(\mathbb{K}_{5}+\mathbb{K}_{6}|x(t)|\right) d z\right) d s \\
\leq & \frac{\kappa(0)}{\beta_{1}}+\frac{\beta_{2}}{\beta_{1}}\left[\frac{\mathbb{L}_{g}}{\Gamma(\vartheta+1)}\left(\delta_{k}-t_{k}\right)^{\vartheta}\right. \\
& \left.+\frac{1}{\Gamma(\vartheta+1)}\left(b-t_{k}\right)^{\vartheta}\left(\mathbb{K}_{2}+\mathbb{K}_{3} \mathcal{M}+\left(\mathbb{K}_{5}+\mathbb{K}_{6} \mathcal{M}\right)\right)\right] \\
& +\frac{1}{\Gamma(\vartheta+1)}(t-s)^{\vartheta}\left(\mathbb{K}_{2}+\mathbb{K}_{3} \mathcal{M}+\mathbb{K}_{5}+\mathbb{K}_{6} \mathcal{M}\right)
\end{aligned}
$$




$$
\begin{aligned}
\leq & \frac{\kappa(0)}{\beta_{1}}+\frac{\beta_{2} \mathbb{L}_{g}}{\beta_{1} \Gamma(\vartheta+1)}\left(\delta_{k}-t_{k}\right)^{\vartheta} \\
& +\left(\frac{\beta_{2}}{\beta_{1} \Gamma(\vartheta+1)}\left(b-t_{k}\right)^{\vartheta}+\frac{1}{\Gamma(\vartheta+1)}(t-s)^{\vartheta}\right)\left(\mathbb{K}_{2}+\mathbb{K}_{3} \mathcal{M}+\mathbb{K}_{5}+\mathbb{K}_{6} \mathcal{M}\right) \\
\leq & \frac{\kappa(0)}{\beta_{1}}+\frac{b^{\vartheta} \beta_{2} \mathbb{L}_{g}}{\beta_{1} \Gamma(\vartheta+1)} \\
& +\left(\frac{b^{\vartheta} \beta_{2}}{\beta_{1} \Gamma(\vartheta+1)}+\frac{b^{\vartheta}}{\Gamma(\vartheta+1)}\right)\left(\mathbb{K}_{2}+\mathbb{K}_{3} \mathcal{M}+\mathbb{K}_{5}+\mathbb{K}_{6} \mathcal{M}\right)
\end{aligned}
$$

Now, for the $t \in\left(\delta_{k}, t_{k+1}\right]$, we have

$$
\begin{aligned}
\|\mathcal{T} x(t)\|= & \| \frac{1}{\Gamma(\vartheta)} \int_{t_{k}}^{\delta_{k}}\left(\delta_{k}-s\right)^{\vartheta-1} \mathcal{G}_{k}\left(s, x\left(s_{k}^{-}\right)\right) d s \\
& +\frac{1}{\Gamma(\vartheta)} \int_{t_{k}}^{t}(t-s)^{\vartheta-1}\left(\Psi(s, x(s))+\int_{a}^{s} \mathcal{K}_{0}(s, z, x(z)) d z\right) d s \| \\
\leq & \frac{1}{\Gamma(\vartheta)} \int_{t_{k}}^{\delta_{k}}\left(\delta_{k}-s\right)^{\vartheta-1} \mathbb{L}_{g} d s \\
& +\frac{1}{\Gamma(\vartheta)} \int_{t_{k}}^{t}(t-s)^{\vartheta-1}\left(\mathbb{K}_{2}+\mathbb{K}_{3}|x(t)|+\int_{a}^{s}\left(\mathbb{K}_{5}+\mathbb{K}_{6}|x(t)|\right) d z\right) d s \\
\leq & \frac{\mathbb{L}_{g}}{\Gamma(\vartheta+1)}\left(\delta_{k}-t_{k}\right)^{\vartheta}+\frac{1}{\Gamma(\vartheta+1)}\left(t-t_{k}\right)^{\vartheta}\left(\mathbb{K}_{2}+\mathbb{K}_{3} \mathcal{M}+\left(\mathbb{K}_{5}+\mathbb{K}_{6} \mathcal{M}\right)\right) \\
\leq & \frac{1}{\Gamma(\vartheta+1)} \mathbb{L}_{g} b^{\vartheta}+\frac{1}{\Gamma(\vartheta+1)} b^{\vartheta}\left(\mathbb{K}_{2}+\mathbb{K}_{3} \mathcal{M}+\left(\mathbb{K}_{5}+\mathbb{K}_{6} \mathcal{M}\right)\right) .
\end{aligned}
$$

Now, for $t \in\left(t_{k}, \delta_{k}\right]$, where $k=1,2,3, \ldots, p$ and $x(t) \in \mathbb{B}$, we have

$$
\begin{aligned}
\|\mathcal{T} x(t)\| & =\left\|\frac{1}{\Gamma(\vartheta)} \int_{t_{k}}^{t}(t-s)^{\vartheta-1} \mathcal{G}_{k}\left(s, x\left(s_{k}^{-}\right)\right) d s\right\| \\
& \leq \frac{1}{\Gamma(\vartheta)} \int_{t_{k}}^{t}(t-s)^{\vartheta-1}\left\|\mathcal{G}_{k}\left(s, x\left(s_{k}^{-}\right)\right)\right\| d s \\
& \leq \frac{\mathbb{L}_{g}}{\Gamma(\vartheta+1)}\left(t-t_{k}\right)^{\vartheta} \\
& \leq \frac{\mathbb{L}_{g}}{\Gamma(\vartheta+1)} b^{\vartheta} .
\end{aligned}
$$

By the help of (3.2)-(3.3), we have $\|\mathcal{T} x\| \leq \chi$. This implies that $\mathcal{T}: \mathbb{B} \rightarrow \mathbb{B}$.

Step 2. Now, we show that $\mathcal{T}$ is a strict contraction. For this, we assume $x(t), y(t) \in \mathbb{R}$. And consider the following three cases.

Case I For $t \in\left[0, t_{1}\right]$, we have

$$
\begin{aligned}
& \|\mathcal{T} x(t)-\mathcal{T} y(t)\| \\
& =\| \frac{\kappa(0)}{\beta_{1}}-\frac{\beta_{2}}{\beta_{1}}\left[\frac{1}{\Gamma(\vartheta)} \int_{t_{k}}^{\delta_{k}}\left(\delta_{k}-s\right)^{\vartheta-1} \mathcal{G}_{k}\left(s, x\left(s_{k}^{-}\right)\right) d s\right. \\
& \left.\quad+\frac{1}{\Gamma(\vartheta)} \int_{t_{k}}^{b}(b-s)^{\vartheta-1}\left(\Psi(s, x(s))+\int_{a}^{s} \mathcal{K}_{0}(s, z, x(z)) d z\right) d z\right]
\end{aligned}
$$




$$
\begin{aligned}
& +\frac{1}{\Gamma(\vartheta)} \int_{0}^{t}(t-s)^{\vartheta-1}\left(\Psi(s, x(s))+\int_{a}^{s} \mathcal{K}_{0}(s, z, x(z)) d z\right) d s \\
& -\left(\frac{\kappa(0)}{\beta_{1}}-\frac{\beta_{2}}{\beta_{1}}\left[\frac{1}{\Gamma(\vartheta)} \int_{t_{k}}^{\delta_{k}}\left(\delta_{k}-s\right)^{\vartheta-1} \mathcal{G}_{k}\left(s, y\left(s_{k}^{-}\right)\right) d s\right.\right. \\
& \left.+\frac{1}{\Gamma(\vartheta)} \int_{t_{k}}^{b}(b-s)^{\vartheta-1}\left(\Psi(s, y(s))+\int_{a}^{s} \mathcal{K}_{0}(s, z, y(z)) d z\right) d z\right] \\
& \left.+\frac{1}{\Gamma(\vartheta)} \int_{0}^{t}(t-s)^{\vartheta-1}\left(\Psi(s, y(s))+\int_{a}^{s} \mathcal{K}_{0}(s, z, y(z)) d z\right) d s\right) \| \\
& \leq \frac{\beta_{2}}{\beta_{1}}\left[\frac{1}{\Gamma(\vartheta)} \int_{t_{k}}^{\delta_{k}}\left(\delta_{k}-s\right)^{\vartheta-1} \mathbb{L}_{g}\|x-y\| d s\right. \\
& +\frac{1}{\Gamma(\vartheta)} \int_{t_{k}}^{b}(b-s)^{\vartheta-1}(\|\Psi(s, x(s))-\Psi(s, y(s))\| \\
& \left.\left.+\int_{a}^{s}\left\|\mathcal{K}_{0}(s, z, x(z))-\mathcal{K}_{0}(s, z, y(z))\right\| d z\right) d z\right] \\
& +\frac{1}{\Gamma(\vartheta)} \int_{0}^{t}(t-s)^{\vartheta-1}(\|\Psi(s, x(s))-\Psi(s, y(s))\| \\
& \left.+\int_{a}^{s}\left\|\mathcal{K}_{0}(s, z, x(z))-\mathcal{K}_{0}(s, z, y(z))\right\| d z\right) d s \\
& \leq \frac{\beta_{2}}{\beta_{1}}\left[\frac{1}{\Gamma(\vartheta)} \int_{t_{k}}^{\delta_{k}}\left(\delta_{k}-s\right)^{\vartheta-1} \mathbb{L}_{g}\|x-y\| d s\right. \\
& \left.+\frac{1}{\Gamma(\vartheta)} \int_{t_{k}}^{b}(b-s)^{\vartheta-1}\left(\mathbb{K}_{1}\|x-y\|+\int_{a}^{s} \mathbb{K}_{4}\|x-y\| d z\right) d z\right] \\
& +\frac{1}{\Gamma(\vartheta)} \int_{0}^{t}(t-s)^{\vartheta-1}\left(\mathbb{K}_{1}\|x-y\|+\int_{a}^{s} \mathbb{K}_{4}\|x-y\| d z\right) d s \\
& \leq\left[\frac{\beta_{2}}{\beta_{1}}\left(\frac{b^{\vartheta}}{\Gamma(\vartheta+1)} \mathbb{L}_{g}+\frac{b^{\vartheta}}{\Gamma(\vartheta+1)}\left(\mathbb{K}_{1}+b \mathbb{K}_{4}\right)\right)\right. \\
& \left.+\frac{b^{\vartheta}}{\Gamma(\vartheta+1)}\left(\mathbb{K}_{1}+b \mathbb{K}_{4}\right)\right]\|x-y\| \text {. }
\end{aligned}
$$

For $t \in\left(\delta_{k}, t_{k+1}\right]$, we have

$$
\begin{aligned}
\|\mathcal{T} x(t)-\mathcal{T} y(t)\| \\
=\| \frac{1}{\Gamma(\vartheta)} \int_{t_{k}}^{\delta_{k}}\left(\delta_{k}-s\right)^{\vartheta-1} \mathcal{G}_{k}\left(s, x\left(s_{k}^{-}\right)\right) d s+\frac{1}{\Gamma(\vartheta)} \int_{t_{k}}^{t}(t-s)^{\vartheta-1}(\Psi(s, x(s)) \\
\left.\quad+\int_{a}^{s} \mathcal{K}_{0}(s, z, x(z)) d z\right) d s-\frac{1}{\Gamma(\vartheta)} \int_{t_{k}}^{\delta_{k}}\left(\delta_{k}-s\right)^{\vartheta-1} \mathcal{G}_{k}\left(s, y\left(s_{k}^{-}\right)\right) d s \\
\quad-\frac{1}{\Gamma(\vartheta)} \int_{t_{k}}^{t}(t-s)^{\vartheta-1}\left(\Psi(s, y(s))-\int_{a}^{s} \mathcal{K}_{0}(s, z, y(z)) d z\right) d s \| \\
\leq \frac{1}{\Gamma(\vartheta)} \int_{t_{k}}^{\delta_{k}}\left(\delta_{k}-s\right)^{\vartheta-1}\left\|\mathcal{G}_{k}\left(s, x\left(s_{k}^{-}\right)\right)-\mathcal{G}_{k}\left(s, y\left(s_{k}^{-}\right)\right)\right\| d s \\
\quad+\frac{1}{\Gamma(\vartheta)} \int_{t_{k}}^{t}(t-s)^{\vartheta-1}(\|\Psi(s, x(s))-\Psi(s, y(s))\|
\end{aligned}
$$




$$
\begin{aligned}
& \left.+\int_{a}^{s}\left\|\mathcal{K}_{0}(s, z, x(z))-\mathcal{K}_{0}(s, z, y(z))\right\| d z\right) d s \\
\leq & {\left[\frac{1}{\Gamma(\vartheta+1)} b^{\vartheta} \mathbb{K}_{g}+\frac{1}{\Gamma(\vartheta+1)} b^{\vartheta}\left(\mathbb{K}_{1}+b \mathbb{K}_{4}\right)\right]\|x-y\| . }
\end{aligned}
$$

Now, for $t \in\left(t_{k}, \delta_{k}\right]$, where $k=1,2,3, \ldots, p$ and $x(t) \in \mathbb{B}$, we have

$$
\begin{aligned}
& \|\mathcal{T} x(t)-\mathcal{T} y(t)\| \\
& \quad=\left\|\frac{1}{\Gamma(\vartheta)} \int_{t_{k}}^{t}(t-s)^{\vartheta-1} \mathcal{G}_{k}\left(s, x\left(s_{k}^{-}\right)\right) d s-\frac{1}{\Gamma(\vartheta)} \int_{t_{k}}^{t}(t-s)^{\vartheta-1} \mathcal{G}_{k}\left(s, y\left(s_{k}^{-}\right)\right) d s\right\| \\
& \quad \leq \frac{1}{\Gamma(\vartheta)} \int_{t_{k}}^{t}(t-s)^{\vartheta-1}\left\|\mathcal{G}_{k}\left(s, x\left(s_{k}^{-}\right)\right)-\mathcal{G}_{k}\left(s, y\left(s_{k}^{-}\right)\right)\right\| d s \\
& \quad \leq \frac{\mathbb{K}_{g}}{\Gamma(\vartheta+1)}\left(t-t_{k}\right)^{\vartheta}\|x-y\| \\
& \leq \frac{\mathbb{K}_{g}}{\Gamma(\vartheta+1)} b^{\vartheta}\|x-y\| .
\end{aligned}
$$

Thus, with the help of (3.5)-(3.7), we have that the operator $\mathcal{T}$ is a contraction, and by the Banach fixed point theorem $\mathcal{T}$ has a unique fixed point. This further implies that fractional impulsive system (1.1) has a unique solution, which accomplishes the proof.

\section{Data dependence}

Here, we present data-dependence of the solution of impulsive system (1.1). We follow the results studied given in $[12,24]$.

Theorem 4.1 Assume that $\left(\mathcal{A}_{1}\right)$ to $\mathcal{A}_{3}$ are satisfied. Then, for $x(t), \widetilde{x}(t)$ satisfying (2.11), we have $\|x(t)-\widetilde{x}(t)\|_{\infty}<\zeta$.

Proof With the help of Theorem 2.1, we have

$$
x(t)-\widetilde{x}(t)=\left\{\begin{array}{c}
\frac{\kappa(0)}{\beta_{1}}-\frac{\beta_{2}}{\beta_{1} \Gamma(\vartheta)}\left[\int_{t_{k}}^{\delta_{k}}\left(\delta_{k}-s\right)^{\vartheta-1} \mathcal{G}_{k}\left(s, x\left(s_{k}^{-}\right)\right) d s\right. \\
\left.\quad+\int_{t_{k}}^{b}(b-s)^{\vartheta-1}\left(\Psi(s, x(s))+\int_{a}^{s} \mathcal{K}_{0}(s, z, x(z)) d z\right) d z\right] \\
\quad+\frac{1}{\Gamma(\vartheta)} \int_{0}^{t}(t-s)^{\vartheta-1}\left(\Psi(s, x(s))+\int_{a}^{s} \mathcal{K}_{0}(s, z, x(z)) d z\right) d s \\
\quad-\frac{\widetilde{\kappa(0)}}{\beta_{1}}+\frac{\beta_{2}}{\beta_{1} \Gamma(\vartheta)}\left[\int_{t_{k}}^{\delta_{k}}\left(\delta_{k}-s\right)^{\vartheta-1} \mathcal{G}_{k}\left(s, \widetilde{x}\left(s_{k}^{-}\right)\right) d s\right. \\
\left.\quad+\int_{t_{k}}^{b}(b-s)^{\vartheta-1}\left(\Psi(s, \widetilde{x}(s))-\int_{a}^{s} \mathcal{K}_{0}(s, z, \widetilde{x}(z)) d z\right) d z\right] \\
\quad-\frac{1}{\Gamma(\vartheta)} \int_{0}^{t}(t-s)^{\vartheta-1}\left(\Psi(s, \widetilde{x}(s))+\int_{a}^{s} \mathcal{K}_{0}(s, z, \widetilde{x}(z)) d z\right) d s, \\
\quad \text { for } t \in\left[0, t_{1}\right], \\
\frac{1}{\Gamma(\vartheta)} \int_{t_{k}}^{\delta_{k}}\left(\delta_{k}-s\right)^{\vartheta-1} \mathcal{G}_{k}\left(s, x\left(s_{k}^{-}\right)\right) d s+\frac{1}{\Gamma(\vartheta)} \int_{t_{k}}^{t}(t-s)^{\vartheta-1}(\Psi(s, x(s)) \\
\left.\quad+\int_{a}^{s} \mathcal{K}_{0}(s, z, x(z)) d z\right) d s-\frac{1}{\Gamma(\vartheta)} \int_{t_{k}}^{\delta_{k}}\left(\delta_{k}-s\right)^{\vartheta-1} \mathcal{G}_{k}\left(s, \widetilde{x}\left(s_{k}^{-}\right)\right) d s \\
\quad-\frac{1}{\Gamma(\vartheta)} \int_{t_{k}}^{t}(t-s)^{\vartheta-1}\left(\Psi(s, \widetilde{x}(s))+\int_{a}^{s} \mathcal{K}_{0}(s, z, \widetilde{x}(z)) d z\right) d s, \\
\quad \text { for } t \in\left[\delta_{k}, t_{k+1}\right], \\
\frac{1}{\Gamma(\vartheta)} \int_{t_{k}}^{t}(t-s)^{\vartheta-1} \mathcal{G}_{k}\left(s, x\left(s_{k}^{-}\right)\right) d s-\frac{1}{\Gamma(\vartheta)} \int_{t_{k}}^{t}(t-s)^{\vartheta-1} \mathcal{G}_{k}\left(s, \widetilde{x}\left(s_{k}^{-}\right)\right) d s, \\
\quad \text { for } t \in\left[t_{k}, \delta_{k}\right] .
\end{array}\right.
$$


Case 1. Then, for $t \in\left[0, t_{1}\right]$, we have

$$
\begin{aligned}
\| x(t) & -\widetilde{x}(t) \|_{\infty} \\
= & \| \frac{\kappa(0)}{\beta_{1}}-\frac{\beta_{2}}{\beta_{1} \Gamma(\vartheta)}\left[\int_{t_{k}}^{\delta_{k}}\left(\delta_{k}-s\right)^{\vartheta-1} \mathcal{G}_{k}\left(s, x\left(s_{k}^{-}\right)\right) d s\right. \\
& \left.+\int_{t_{k}}^{b}(b-s)^{\vartheta-1}\left(\Psi(s, x(s))+\int_{a}^{s} \mathcal{K}_{0}(s, z, x(z)) d z\right) d z\right] \\
& +\frac{1}{\Gamma(\vartheta)} \int_{0}^{t}(t-s)^{\vartheta-1}\left(\Psi(s, x(s))+\int_{a}^{s} \mathcal{K}_{0}(s, z, x(z)) d z\right) d s \\
& -\frac{\kappa(0)}{\beta_{1}}+\frac{\beta_{2}}{\beta_{1} \Gamma(\vartheta)}\left[\int_{t_{k}}^{\delta_{k}}\left(\delta_{k}-s\right)^{\vartheta-1} \mathcal{G}_{k}\left(s, \widetilde{x}\left(s_{k}^{-}\right)\right) d s\right. \\
& \left.+\int_{t_{k}}^{b}(b-s)^{\vartheta-1}\left(\Psi(s, \widetilde{x}(s))-\int_{a}^{s} \mathcal{K}_{0}(s, z, \widetilde{x}(z)) d z\right) d z\right] \\
& -\frac{1}{\Gamma(\vartheta)} \int_{0}^{t}(t-s)^{\vartheta-1}\left(\Psi(s, \widetilde{x}(s))+\int_{a}^{s} \mathcal{K}_{0}(s, z, \widetilde{x}(z)) d z\right) d s \| \\
\leq & \frac{\|\kappa(0)-\widetilde{\kappa}(0)\|}{\beta_{1}}-\frac{\beta_{2}}{\beta_{1} \Gamma(\vartheta)}\left[\int_{t_{k}}^{\delta_{k}}\left(\delta_{k}-s\right)^{\vartheta-1}\left\|\mathcal{G}_{k}\left(s, x\left(s_{k}^{-}\right)\right)-\mathcal{G}_{k}\left(s, \widetilde{x}\left(s_{k}^{-}\right)\right)\right\| d s\right. \\
& \left.+\int_{a}^{s}\left\|\mathcal{K}_{0}(s, z, x(z))-\mathcal{K}_{0}(s, z, \widetilde{x}(z))\right\| d z\right) d s \\
& +\int_{t_{k}}^{b}(b-s)^{\vartheta-1}(\|\Psi(s, x(s))-\Psi(s, \widetilde{x}(s))\| \\
& \left.\left.+\int_{a}^{s}\left\|\mathcal{K}_{0}(s, z, x(z))-\mathcal{K}_{0}(s, z, \widetilde{x}(z))\right\| d z\right) d z\right]
\end{aligned}
$$

Similarly, for $t \in\left(\delta_{k}, t_{k+1}\right]$, we have

$$
\begin{aligned}
\| x(t) & -\widetilde{x}(t) \|_{\infty} \\
= & \| \frac{1}{\Gamma(\vartheta)} \int_{t_{k}}^{\delta_{k}}\left(\delta_{k}-s\right)^{\vartheta-1} \mathcal{G}_{k}\left(s, x\left(s_{k}^{-}\right)\right) d s+\frac{1}{\Gamma(\vartheta)} \int_{t_{k}}^{t}(t-s)^{\vartheta-1}(\Psi(s, x(s)) \\
& \left.+\int_{a}^{s} \mathcal{K}_{0}(s, z, x(z)) d z\right) d s-\frac{1}{\Gamma(\vartheta)} \int_{t_{k}}^{\delta_{k}}\left(\delta_{k}-s\right)^{\vartheta-1} \mathcal{G}_{k}\left(s, \widetilde{x}\left(s_{k}^{-}\right)\right) d s \\
& -\frac{1}{\Gamma(\vartheta)} \int_{t_{k}}^{t}(t-s)^{\vartheta-1}\left(\Psi(s, \tilde{x}(s))+\int_{a}^{s} \mathcal{K}_{0}(s, z, \widetilde{x}(z)) d z\right) d s \| \\
\leq & \frac{1}{\Gamma(\vartheta)} \int_{t_{k}}^{\delta_{k}}\left(\delta_{k}-s\right)^{\vartheta-1}\left\|\mathcal{G}_{k}\left(s, x\left(s_{k}^{-}\right)\right)-\mathcal{G}_{k}\left(s, \tilde{x}\left(s_{k}^{-}\right)\right)\right\| d s \\
& +\frac{1}{\Gamma(\vartheta)} \int_{t_{k}}^{t}(t-s)^{\vartheta-1}(\|\Psi(s, x(s))-\Psi(s, x(s))\|
\end{aligned}
$$




$$
\begin{aligned}
& \left.+\left\|\int_{a}^{s} \mathcal{K}_{0}(s, z, x(z))-\mathcal{K}_{0}(s, z, x(z))\right\| d z\right) d s \\
\leq & \frac{1}{\Gamma(\vartheta+1)} b^{\vartheta} \mathbb{K}_{g}\|x-\widetilde{x}\|+\frac{1}{\Gamma(\vartheta+1)} b^{\vartheta}\left(\mathbb{K}_{1}\|x-\tilde{x}\|+b \mathbb{K}_{4}\|x-\tilde{x}\|\right) \\
= & \frac{b^{\vartheta}}{\Gamma(\vartheta+1)}\left[\mathbb{K}_{g}+\left(\mathbb{K}_{1}+b \mathbb{K}_{4}\right)\right]\|x-\tilde{x}\| .
\end{aligned}
$$

Finally, for $t \in\left[t_{k}, \delta_{k}\right]$, we have

$$
\begin{aligned}
& \|x(t)-\tilde{x}(t)\|_{\infty} \\
& =\left\|\frac{1}{\Gamma(\vartheta)} \int_{t_{k}}^{t}(t-s)^{\vartheta-1} \mathcal{G}_{k}\left(s, x\left(s_{k}^{-}\right)\right) d s-\frac{1}{\Gamma(\vartheta)} \int_{t_{k}}^{t}(t-s)^{\vartheta-1} \mathcal{G}_{k}\left(s, \widetilde{x}\left(s_{k}^{-}\right)\right) d s\right\| \\
& \leq \frac{1}{\Gamma(\vartheta)} \int_{t_{k}}^{t}(t-s)^{\vartheta-1}\left\|\mathcal{G}_{k}\left(s, x\left(s_{k}^{-}\right)\right)-\mathcal{G}_{k}\left(s, \widetilde{x}\left(s_{k}^{-}\right)\right)\right\| d s \\
& \leq \frac{1}{\Gamma(\vartheta+1)} b^{\vartheta} \mathbb{K}_{g}\|x-\widetilde{x}\| .
\end{aligned}
$$

Thus, by the help of (4.2)-(4.4), $\|x-\widetilde{x}(t)\| \leq \zeta$.

\section{Application}

In order to give verification of the existence and data dependence theorems, here we give the following illustrative model.

Example 5.1 Assume that $\vartheta=0.5, \beta_{1}=5, \beta_{2}=2, b=0.5, I=[0,1]$ and

$$
\left\{\begin{array}{l}
{ }^{c} \mathcal{D}_{0}^{\vartheta} x(t)=\frac{1}{100}\left(\cos (t)+\int_{0}^{t} \sin x(t) d t\right), \quad t \in[0,1], t \neq \frac{k \pi}{2}, \\
x(t)=\frac{\sin (x(t))}{7}, \quad \text { for } t \in\left(t_{k}, \delta_{k}\right] \\
\beta_{1} x(0)+\beta_{2} x(b)=\frac{1}{100} .
\end{array}\right.
$$

Then from (5.1) one can easily evaluate from $\mathcal{M}<\frac{1}{7}, b=1, a=0, \mathbb{K}_{1}=1 / 100, \mathbb{K}_{5}=\mathbb{K}_{2}=0$, $\mathbb{K}_{3}=1 / 100=\mathbb{K}_{6}, \mathbb{K}_{g}=\mathbb{L}_{g}=\frac{1}{7}, \chi<1$. Thus, by the help of Theorem 3.1, system (5.1) has a unique solution $[0,1]$.

\section{Conclusion}

In this article we have considered a fractional order impulsive IDE (1.1) for the existence of unique solution, data dependence, and stability results. We have used basic results from the fixed point theory and literature for fractional order calculus. The results are examined with the help of an expressive numerical example.

Acknowledgements

The authors are very obliged to the editor and referees for their supportive input and useful feedback.

Funding

This research was funded by the Deanship of Scientific Research at Princess Nourah bint Abdulrahman University through the Fast-track Research Funding Program. 


\section{Competing interests}

The authors have no competing interests regarding the publication of this article.

\section{Authors' contributions}

All the authors have equal contributions in this article. All authors read and approved the final manuscript.

\section{Author details}

'Department of Mathematics, Shaheed Benazir Bhutto University Sheringa, Dir Upper, 18000 Khybar Pakhtunkhwa,

Pakistan. ${ }^{2}$ Department of Mathematics, College of Science, Princess Nourah bint Abdulrahman University, Riyadh, Saudi Arabia. ${ }^{3}$ Department of Mathematics, University of Sistan and Baluchestan, Zahedan, Iran. ${ }^{4}$ Department of Mathematics and General Sciences, Prince Sultan University, 66833, 11586 Riyadh, Saudi Arabia.

\section{Publisher's Note}

Springer Nature remains neutral with regard to jurisdictional claims in published maps and institutional affiliations.

\section{Received: 29 April 2020 Accepted: 7 July 2020 Published online: 03 September 2020}

\section{References}

1. Samko, S.G., Kilbas, A.A., Marichev, O.I.: Fractional Integrals and Derivatives: Theory and Applications. Gordon \& Breach, Yverdon (1993)

2. Podlubny, I.: Fractional Differential Equations. Academic Press, New York (1999)

3. Hilfer, R. (ed.): Application of Fractional Calculus in Physics World Scientific, Singapore (2000)

4. Khan, A., Abdeljawad, T., Gomez-Aguilar, J.F., Khan, H.: Dynamical study of fractional order mutualism parasitism food web module. Chaos Solitons Fractals 134, 109685 (2020)

5. Khan, A., Gomez-Aguilar, J.F., Abdeljawad, T., Khan, H.: Stability and numerical simulation of a fractional order plant nectar pollinator model. Alex. Eng. J. 59, 49-59 (2020)

6. Khan, H., Gomez-Aguilar, J.F., Alkhazzan, A., Khan, A.: A fractional order HIV-TB coinfection model with nonsingular Mittag-Leffler law. Math. Methods Appl. Sci. 43(6), 3786-3806 (2020)

7. Khan, A., Gomez-Aguilar, J.F., Khan, T.S., Khan, H.: Stability analysis and numerical solutions of fractional order HIV/AIDS model. Chaos Solitons Fractals 122, 119-128 (2019)

8. Caputo, M., Fabrizio, M.: A new definition of fractional derivative without singular kernel. Prog. Fract. Differ. Appl. 1(2), 73-85 (2015)

9. Abdeljawad, T., Baleanu, D.: On fractional derivatives with exponential kernel and their discrete versions. Rep. Math. Phys. 80(1), 11-27 (2017)

10. Abdeljawad, T.: Fractional operators with exponential kernels and a Lyapunov type inequality. Adv. Differ. Equ. 2017, $313(2017)$

11. Khan, H., Tunc, C., Khan, A.: Green function's properties and existence theorems for nonlinear singular-delay-fractional differential equations. Discrete Contin. Dyn. Syst., Ser. S 13(9), 2475-2487 (2020)

12. Khan, H., Khan, A., Jarad, F., Shah, A.: Existence and data dependence theorems for solutions of an ABC-fractional order impulsive system. Chaos Solitons Fractals 131, 109477 (2020)

13. Khan, H., Khan, A., Abdeljawad, T., Alkhazzan, A.: Existence results in Banach space for a nonlinear impulsive system. Adv. Differ. Equ. 2019, 18 (2019)

14. Khan, A., Khan, H., Gomez-Aguilar, J.F., Abdeljawad, T.: Existence and Hyers-Ulam stability for a nonlinear singular fractional differential equations with Mittag-Leffler kernel. Chaos Solitons Fractals 127, 422-427 (2019)

15. Khan, H., Jarad, F., Abdeljawad, T., Khan, A.: A singular ABC-fractional differential equation with p-Laplacian operator. Chaos Solitons Fractals 129, 56-61 (2019)

16. Sousa, J.V., Kucche, K.D., De Oliveira, E.C.: Stability of psi-Hilfer impulsive fractional differential equations. Appl. Math. Lett. 88, 73-80 (2019)

17. Xu, L., Liu, W.: Ultimate boundedness of impulsive fractional delay differential equations. Appl. Math. Lett. 79, 58-66 (2018)

18. Zhang, T.W., Xiong, L.L.: Periodic motion for impulsive fractional functional differential equations with piecewise Caputo derivative. Appl. Math. Lett. 101, 106072 (2020)

19. Zhao, Y., Chen, H., Xu, C.: Nontrivial solutions for impulsive fractional differential equations via Morse theory. Appl. Math. Comput. 307, 170-179 (2017)

20. Heidarkhani, S., Cabada, A., Afrouzi, G.A., Moradi, S., Caristi, G.: A variational approach to perturbed impulsive fractional differential equations. J. Comput. Appl. Math. 341, 42-60 (2018)

21. Dzhumabaev, D.S.: New general solutions to linear Fredholm integro-differential equations and their applications on solving the boundary value problems. J. Comput. Appl. Math.. 327, 79-108 (2018)

22. Iwankiewicz, R.: Integro-differential Chapman-Kolmogorov equation for continuous-jump Markov processes and its use in problems of multi-component renewal impulse process excitations. Probab. Eng. Mech., 26, 16-25 (2011)

23. Hu, H., Wang, K., Wu, D.: Permanence and global stability for nonautonomous $\mathrm{N}$-species Lotka-Volterra competitive system with impulses and infinite delays. J. Math. Anal. Appl. 377(1), 145-160 (2011)

24. Wang, J., Lv, L., Zhou, Y.: Ulam stability and data dependence for fractional differential equations with Caputo derivative. Electron. J. Qual. Theory Differ. Equ. 201163 (2011) 